
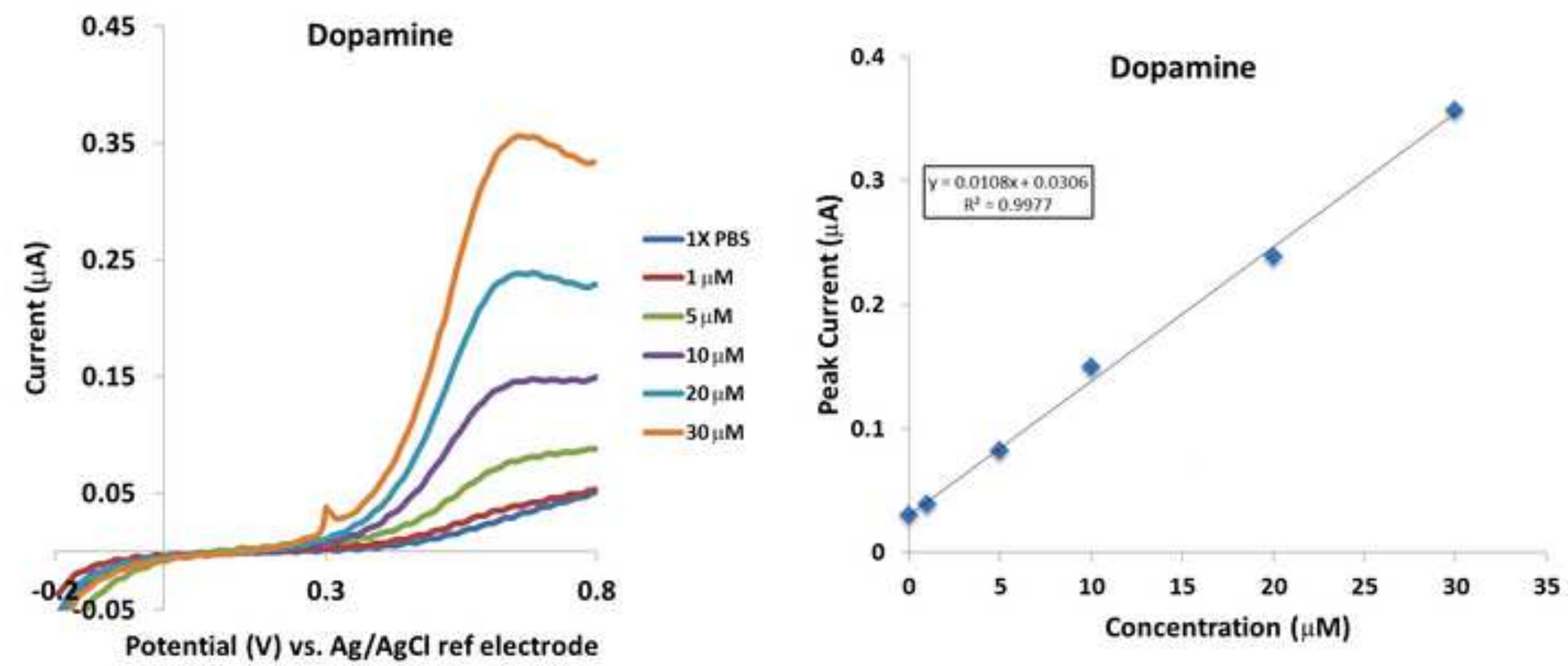


\section{Nitrogen-incorporated Ultrananocrystalline Diamond Microneedle Arrays for Electrochemical Biosensing}

Shelby A. Skoog ${ }^{1}$, Philip R. Miller ${ }^{1,2}$, Ryan D. Boehm ${ }^{1}$, Anirudha V. Sumant ${ }^{3}$, Ronen Polsky ${ }^{2}$, Roger J. Narayan ${ }^{1}$

${ }^{1}$ Joint Dept. of Biomedical Engineering, UNC-Chapel Hill and NC State University, Raleigh, NC

${ }^{2}$ Department of Biosensors and Nanomaterials, Sandia National Laboratories, Albuquerque, NM

${ }^{3}$ Center for Nanoscale Materials, Argonne National Laboratory, Argonne, IL

Corresponding author:

Roger J. Narayan

Tel: 19196968488

Fax: 15096968481

Email: roger narayan@msn.com

Postal address: Joint Department of Biomedical Engineering, University of North Carolina and North Carolina State University, 911 Oval Drive, Box 7115, Raleigh, NC 27695-7715 USA 
Microneedles are minimally invasive transdermal medical devices that are utilized for various applications, including drug delivery, fluid sampling, micro-dialysis, and electrochemical sensing. These devices are associated with less pain and tissue damage as compared with conventional hypodermic needle-based devices. In this study, we demonstrate fabrication of titanium alloy microneedle arrays with nitrogen-incorporated ultrananocrystalline diamond (N-UNCD) coatings. Microneedles were micromachined from ASTM F136 ELI Ti-6Al$4 \mathrm{~V}$ alloy, a widely used medical-grade titanium alloy. N-UNCD coatings were deposited on the microneedles using microwave plasma enhanced chemical vapor deposition to enhance mechanical strength, increase hardness, improve biocompatibility, and provide an electrochemically stable surface. The structural and chemical properties of the N-UNCD titanium alloy microneedle arrays were evaluated using scanning electron microscopy and Raman spectroscopy. The mechanical robustness and skin penetration capability of the devices were demonstrated using cadaveric porcine skin. Finally, the electrochemical properties of the N-UNCD electrodes were evaluated; in vitro electrochemical detection of uric acid and dopamine was demonstrated using unmodified N-UNCD electrodes. These results demonstrate the application potential of N-UNCD-coated titanium alloy microneedles for transdermal electrochemical biosensing applications.

\section{Keywords}

nanocrystalline diamond, microneedles, electrochemistry, biosensor 


\section{Introduction}

In vivo biosensors enable real-time and continuous detection of physiologically relevant molecules for monitoring patient health; as such, these devices are of significant interest to the biomedical community. ${ }^{1}$ The development of electrochemical microneedle-based sensors may allow for minimally invasive transdermal sensing of biomolecules with reduced tissue damage and minimized pain. ${ }^{2}$ These devices use microneedles to access biological fluids and integrated electrodes for highly sensitive, selective electrochemical detection of biomolecules.

Although more extensively evaluated for drug delivery applications, microneedle arrays have been successfully integrated with electrochemical biosensors for analytical sensing of physiologically relevant molecules. For example, glucose sensors have been developed that contain microneedles in conjunction with sampling mechanisms and enzyme-based sensing substrates..$^{3-6}$ Hollow microneedle arrays fabricated by stereolithography have been integrated with carbon fiber and carbon paste electrodes for detection of ascorbic acid, hydrogen peroxide, glucose, glutamate, and lactate. ${ }^{1,2,7,8}$ For example, Windmiller et al. used pyramidal-shaped hollow microneedle arrays and carbon paste electrodes for selective monitoring of lactate, demonstrating lactate detection in the presence of common physiological interferents such as ascorbic acid, uric acid, and acetaminophen. ${ }^{7}$ Miller et al. developed a multiplexed, hollow microneedle-based biosensor array for simultaneous detection of $\mathrm{pH}$, glucose, and lactate to monitor exercise-induced metabolic acidosis and tumor microenvironments. ${ }^{8}$ These microneedle-based sensors provide an attractive platform for minimally invasive and real-time biosensing of several analytes. Clinical translation of these microdevices for in vivo physiological monitoring will require ease of microneedle fabrication, mechanically robust and biocompatible sensor components, as well as electrochemically sensitive and stable electrodes.

Diamond is an extremely stable material that exhibits excellent biocompatibility, chemical inertness, and mechanical robustness. ${ }^{9}$ Advances in thin film technology have enabled deposition of nanocrystalline diamond thin films, which have been considered for use as a biointerface in many types of medical devices. ${ }^{9-11}$ Synthesis of highly conducting NCD thin films through doping has led to use of doped NCD thin films for several electrical and electrochemical applications. ${ }^{12}$ Doped diamond electrodes have demonstrated superior electrochemical properties with low background currents, wide potential windows, high current density electrolysis, and high over potential 
for oxygen evolution. ${ }^{13-17}$ For biosensing applications, diamond electrodes have exhibited low detection limits as well as excellent precision and response stability for detection of numerous analytes without surface modification. ${ }^{16,18}$ Non-functionalized diamond electrodes have been applied for electrochemical detection of numerous physiologically relevant molecules, including acetaminophen ${ }^{19}$, adenosine ${ }^{20}$, ascorbic acid ${ }^{19,21}$, caffeine $^{22}$, chlorpromazine ${ }^{21}$, cysteine $^{23}$, dopamine ${ }^{24-25}$, glutathione ${ }^{26}$, histamine $^{27}$, nicotinamide adenine dinucleotide $(\mathrm{NADH})^{24}$, oxalic acid ${ }^{28}$, and serotonin..$^{27,29}$

In addition to their superior electrochemical properties, conductive diamond electrodes may function in the harsh, electrolytic physiological environment without degradation, unlike other microelectronic materials such as silicon, $\mathrm{SiO}_{\mathrm{x}}$, and gold. ${ }^{30}$ The chemical stability and inertness of diamond facilitates its use for biological applications. Furthermore, low adsorption of proteins and contaminants by diamond reduces fouling, enabling extended in vivo use of diamond electrodes. ${ }^{16}$

In this study, we demonstrate fabrication of titanium alloy microneedle arrays with nitrogen-incorporated ultrananocrystalline diamond (N-UNCD) coatings. The integration of conductive nanocrystalline diamond on a microneedle platform enables minimally invasive, highly stable electrochemical biosensing of transdermal fluids. Microneedle arrays were machined from a medical-grade titanium alloy and coated with nitrogen-incorporated ultrananocrystalline diamond using microwave plasma chemical vapor deposition. The N-UNCD coating provides a biocompatible interface with an electrochemically stable surface. The structural and chemical properties of the $\mathrm{N}$ UNCD-coated titanium alloy microneedle arrays were examined using scanning electron microscopy and Raman spectroscopy. The microneedle arrays were evaluated for their mechanical robustness and skin penetration capabilities using a mechanical testing instrument. Finally, the electrochemical properties of the N-UNCD electrodes were evaluated; in vitro electrochemical detection of uric acid and dopamine at physiologically relevant concentrations was examined using unmodified N-UNCD electrodes. To our knowledge, this is the first development of nitrogen-incorporated ultrananocrystalline diamond microneedle arrays for transdermal electrochemical biosensing applications. The results suggest that N-UNCD microneedle-based device may serve as an attractive platform for minimally invasive, continuous monitoring of physiologically relevant molecules.

\section{Materials \& Methods}




\subsection{Fabrication of Nitrogen-incorporated Ultrananocrystalline Diamond-coated Titanium Alloy Microneedle Arrays}

Microneedle arrays were micromachined from a medical-grade ASTM F136 ELI Ti-6AI-4V alloy bar (Allegheny Technologies, Pittsburgh, PA) using 3-axis computer numerical control (CNC) milling. The titanium microneedle arrays were fabricated with 7 microneedles in a staggered orientation. The solid microneedles were conical in shape and exhibited heights of approximately $340 \mu \mathrm{m}$ and base diameters of approximately $230 \mu \mathrm{m}$. Titanium alloy disks with diameters of $1.6 \mathrm{~cm}$ and thicknesses of $2.5 \mathrm{~mm}$ were also fabricated to provide flat substrates for electrochemical testing.

Nitrogen-incorporated ultrananocrystalline diamond was deposited on the titanium alloy microneedle arrays and titanium alloy disks using a $915 \mathrm{MHz}$ microwave plasma chemical vapor deposition (MPCVD) (Lambda Technologies, Raleigh, NC) facility located at Argonne National Laboratory. To clean the titanium alloy substrates, samples were sonicated in acetone and subsequently rinsed in methanol to remove debris and contaminants. Prior to N-UNCD deposition, the titanium alloy substrates underwent nucleation pretreatment via sonication in nanodiamond suspension in methanol for 20 minutes with subsequent rinsing steps in acetone, isopropanol, and methanol. The titanium alloy samples were then adhered to a silicon wafer with minimal silver paste before loading into the CVD chamber. N-UNCD deposition was conducted over a deposition time of one hour; a gas mixture of argon:methane:nitrogen at a ratio of $160 \mathrm{sccm}: 3 \mathrm{sccm}: 40 \mathrm{sccm}$ was used in this study. The deposition was conducted at a substrate temperature of $850^{\circ} \mathrm{C}$, a working pressure of $80 \mathrm{mbar}$, and an input power of 2500 W.

\subsection{Physico-Chemical Characterization of Uncoated and N-UNCD-Coated Microneedles and Disks}

Uncoated and N-UNCD-coated titanium alloy microneedle arrays and disks were evaluated using scanning electron microscopy (SEM). The surface morphology and microneedle structure were examined using a Nova 600 NanoLab dual-beam scanning electron microscope/focused ion beam instrument (FEI, Hillsboro, OR). Image acquisition was performed using an acceleration voltage of $20 \mathrm{keV}$ and a current of $0.62 \mathrm{nA}$.

Confocal Raman microscopy was conducted on the N-UNCD coatings with a Renishaw inVia Raman spectrometer (Renishaw, Gloucestershire, UK) in extended mode and a He-Ne laser with a wavelength of $633 \mathrm{~nm}$. 
The laser was introduced to the sample through a 50X objective lens. For microneedle arrays, Raman spectra were obtained at both the microneedle tip and the flat base of the substrate.

\subsection{Porcine Skin Penetration Testing of N-UNCD Microneedles}

Microneedle arrays were imaged with a Leica EZ4 D stereo microscope (Leica Microsystems, Wetzlar, Germany) before and after insertion into cadaveric porcine skin. Excised, full-thickness cadaveric porcine skin was obtained from a local abattoir and cut to $4.5 \mathrm{~cm} \times 4.5 \mathrm{~cm}$. The cadaveric porcine skin was inserted into a customized tissue holder to maintain tension in the tissue during penetration testing. The customized tissue holder was screwed into the base fixture of a Bose Electroforce ${ }^{\circledR} 3100$ mechanical testing instrument (Bose Corporation, Framingham, MA) (Figure 3A). Using double-sided adhesive tape, a single microneedle array was fixed to the top platen of the instrument (Figure 3A). The microneedle array was placed in contact with the porcine skin to a load of $0.2 \mathrm{~N}$ prior to testing. The microneedle array was lowered onto the skin at a rate of $0.1 \mathrm{~mm} / \mathrm{s}$ to a load limit of 7 N.

After withdrawal of the microneedle array, the cadaveric porcine skin was dyed with a few droplets of 1 $\mathrm{mg} / \mathrm{mL}$ methylene blue dye for 20 minutes to stain the penetrated tissue. Excess dye was then removed by gently wiping the skin surface with sterile saline swabs followed by ethanol swabs. Subsequently, the stained tissue was observed under a Leica EZ4 D stereo microscope and the penetration holes were imaged.

Following porcine skin penetration testing, the N-UNCD microneedle arrays were imaged using SEM to determine if the microneedles were damaged during insertion. Secondary electron images (SEI) of the microneedle arrays were obtained using a JEOL JSM-6390 HV scanning electron microscope (JEOL, Tokyo, Japan). An acceleration voltage of $15 \mathrm{keV}$ and a working distance of $11 \mathrm{~mm}$ were used for image acquisition.

\subsection{Electrochemical Characterization of N-UNCD Electrodes}

Nitrogen-incorporated ultrananocrystalline diamond-coated titanium and uncoated titanium substrates were cleaned prior to electrochemical measurements by sonicating in ethanol for 10 minutes with subsequent rinsing in nanopure DI water and drying with nitrogen. Initial electrochemical characterization of the electrodes was performed using cyclic voltammograms in $5 \mathrm{mM}$ potassium ferricyanide in $0.1 \mathrm{M} \mathrm{KCl}$ aqueous solution on a Model 273A Potentiostat/Galvanostat (Princeton Applied Research, Oak Ridge, TN). A platinum wire auxiliary electrode 
served as the counter electrode and an external $\mathrm{Ag} / \mathrm{AgCl}(3 \mathrm{M} \mathrm{NaCl})$ electrode was used as the reference electrode. The N-UNCD coated titanium alloy disk working electrode was covered with polyethylene cleanroom tape (ITW $\operatorname{ALMA}^{\mathrm{TM}}$, ThermoFisher Scientific, Waltham, MA) containing a $1 / 8$ inch diameter exposed active area $\left(8 \mathrm{~mm}^{2}\right)$. Cyclic voltammetric scans were performed from $-0.1 \mathrm{~V}$ to $0.6 \mathrm{~V}$ at scan rates ranging from $10-300 \mathrm{mV} / \mathrm{s}$.

\subsection{Electrochemical Detection of Uric Acid and Dopamine Using Unmodified N-UNCD Electrodes}

For electrochemical detection of uric acid and dopamine, the N-UNCD working electrodes were cleaned as described above. The working area of the electrode was controlled by laser cutting (Universal Laser Systems, Scottsdale, AZ) 1/8 inch diameter circles into electroplating tape and applying the tape to the N-UNCD electrodes by hand. Patterns for the working area were drawn in CorelDraw (Corel Corporation, Ottawa, Canada) and imported into the Universal Laser Systems software with laser cutting parameters of $100 \%$ power, $75 \%$ write speed, $300 \mathrm{DPI}$, and 0.0 inch stage height. Detection of uric acid and dopamine was made using linear sweep voltammetry (LSV) with a commercial potentiostat (DropSens, Llanera, Spain). Scans were conducted from $-0.2 \mathrm{~V}$ to $0.8 \mathrm{~V}$ at a scan rate of $10 \mathrm{mV} / \mathrm{s}$. Dopamine concentrations were varied from $1 \mu \mathrm{M}$ to $30 \mu \mathrm{M}$ and uric acid concentrations were varied from $25 \mu \mathrm{M}$ to $200 \mu \mathrm{M}$. All electrochemical measurements were made against an $\mathrm{Ag} / \mathrm{AgCl}$ reference and platinum wire counter electrode.

\section{Results \& Discussion}

\subsection{Fabrication and Characterization of N-UNCD coated Titanium Alloy Microneedles}

The development of a microneedle-based electrode would enable minimally invasive transdermal detection of biomolecules. These devices use microscale needles to access interstitial fluid, which contains biomolecules that can be used to monitor patient health and/or diagnose medical conditions; for example, continuous glucose sensing may be used to improve control over blood glucose levels in diabetes mellitus patients. Microneedle-based platforms eliminate the stress and pain associated with application of conventional hypothermic needles and do not require a trained medical professional for use. ${ }^{2}$ Furthermore, microneedles can access biological fluids without the interference of perspiration or skin irritation, which may serve as limitations for noninvasive transdermal sensors ${ }^{31}$. Microneedle arrays may also be created that enable simultaneous detection of a variety of analytes (i.e., multiplexed biosensing). ${ }^{8}$ As such, microneedle-based electrodes may be utilized for real- 
time and continuous detection of physiologically relevant molecules for evaluating patient health in a rapid and straightforward manner.

Ti-6Al-4V is a titanium alloy that is widely used in biomedical applications, including orthopedic implants, dental screws, dental implants, and cardiovascular devices. ${ }^{32}$ ASTM F136 ELI Ti-6Al-4V alloy was chosen as the microneedle substrate material due to its biocompatibility, mechanical robustness, and ease of fabrication. Furthermore, Ti-6Al-4V has been previously shown to be a suitable substrate material for deposition of nanocrystalline diamond thin films. ${ }^{33}$

Titanium alloy microneedle arrays were successfully fabricated in a reproducible manner using 3-axis CNC milling. The uncoated titanium alloy microneedle arrays showed minimal variation in microneedle dimensions and in spatial orientation. The microneedles were arranged in two rows in a staggered orientation with horizontal center-to-center spacing of $1.4 \mathrm{~mm}$ and diagonal center-to-center spacing of $1 \mathrm{~mm}$. The conical microneedles exhibited heights of approximately $340 \mu \mathrm{m}$ and base diameters of approximately $230 \mu \mathrm{m}$, giving an aspect ratio of approximately 1.5 . The microneedle tip diameters measured approximately $10-20 \mu \mathrm{m}$. Microneedle heights of a few hundred micrometers were chosen to enable penetration of the epidermis of human skin (50-150 $\mu \mathrm{m})$ but preclude interaction with nerve receptors in the deeper layers of the tissue. ${ }^{34}$ High magnification SEM images show no significant defects; the rough surface topology and the visible striations, grooves, and pits on the surface were attributed to the milling process (Figure $1 \mathrm{~A}-\mathrm{C}$ ).

Nanocrystalline diamond was chosen as the electrode material since it is a stable and chemically inert substrate that exhibits mechanical robustness and excellent biocompatibility. Nanocrystalline diamond has been widely considered for use in electrochemical biosensing applications due to its advantageous electrochemical properties such as a wide band gap and a large electrochemical potential window. ${ }^{35-36}$ The surface chemistry of nanocrystalline diamond enables functionalization to produce an exceptionally tunable biomolecular interface. ${ }^{37} \mathrm{~A}$ comparison of DNA-modified UNCD with commonly used substrates for biological functionalization (e.g., gold, glass, glassy carbon, and silicon) demonstrated that UNCD provides higher stability and sensitivity than the other substrates, making it an ideal substrate for biological modification and use in biosensing. ${ }^{38}$ Furthermore, the mechanical robustness and chemical resilience of nanocrystalline diamond makes nanocrystalline diamond 
electrodes less susceptible to physical and chemical degradation in the harsh physiological environment, permitting use in long-term sensing applications.

To synthesize an electrically conductive diamond film, a dopant is incorporated within the diamond film during the CVD deposition process. Nanocrystalline diamond may be doped with boron to create a p-type semiconductor or nitrogen to create a n-type semiconductor. ${ }^{39}$ Nitrogen incorporation during growth leads to enhanced $\mathrm{sp}^{2}$-bonded carbon regions at the grain boundaries of the UNCD film, resulting in improved electrical conductivity. ${ }^{40-41}$ Compared to boron-doped diamond, nitrogen-doped nanocrystalline diamond shows a wide working potential window and a minimized background current. ${ }^{10} \mathrm{~N}-\mathrm{UNCD}$ films have been used for electrochemical sensing of several important physiologically relevant molecules, such as glucose ${ }^{10}$ and dopamine. $^{25,42} \mathrm{Xu}$ et al. used nitrogen-doped diamond electrodes for amperometric detection of glucose, demonstrating glucose detection with good temperature stability and with long-term stability. ${ }^{10}$ Shalini et al. demonstrated detection of dopamine in the presence of common biological interferences (e.g., ascorbic acid and uric acid). ${ }^{25,42}$

In our study, nitrogen-incorporated ultrananocrystalline diamond films were deposited on the titanium alloy microneedle arrays and titanium alloy disks using MPCVD. The thickness of the electrically conductive NUNCD film that was deposited for one hour was approximately $350 \mathrm{~nm}$. SEM micrographs of the N-UNCD coating on the titanium substrates show a uniform, conformal N-UNCD coating with small grain sizes (Figure 1D-F). The NUNCD film deposited was densely packed and continuous, even on the microneedle tips that contained grooves and pits.

Confocal Raman spectra of the N-UNCD coatings are shown in Figure 2. Raman spectra were obtained on the N-UNCD coated microneedles at the microneedle tip and at the flat base of substrate to confirm the chemical bonding at these locations. Raman spectra were also obtained on the N-UNCD coated titanium alloy disks to ensure the chemical bonding of the films on titanium alloy disks was similar that on the microneedles. Raman spectra of the microneedle tip, flat substrate of the microneedle, and the N-UNCD coated disk all exhibited the same three primary peaks. The broad shoulder observed at $1140 \mathrm{~cm}^{-1}$ is associated with transpolyacetylene at the grain boundaries of the nanocrystalline diamond film. ${ }^{43}$ The D-band peak observed at $1340 \mathrm{~cm}^{-1}$ and the G-band 
peak observed around $1580 \mathrm{~cm}^{-1}$ are both attributed to $\mathrm{sp}^{2}$-bonded carbon. Typically, the G-band peak is observed closer to $1550 \mathrm{~cm}^{-1}$; however, a shift in the position of the G-band to higher wavenumbers has been observed in nitrogen-incorporated UNCD films due to a slight variation in the configuration of the $\mathrm{sp}^{2}$-bonded carbon at the grain boundaries. ${ }^{44-45}$ The flat base of the microneedle substrate and the coated disk exhibit similar ratios between the D-band peak and the G-band peak. This ratio is slightly higher than that for the microneedle tip, potentially due to differences in film growth from temperature variation within the device during deposition. Birrell et al. observed an increase in this ratio as increasing concentrations of nitrogen were incorporated into the plasma during NCD deposition. $^{45}$

\subsection{Porcine Skin Penetration Testing of N-UNCD Microneedles}

Microneedle arrays were applied to cadaveric porcine skin at a rate of $0.1 \mathrm{~mm} / \mathrm{s}$ to a load limit of $7 \mathrm{~N}$ using a Bose Electroforce ${ }^{\circledR} 3100$ mechanical test instrument, as shown in Figure 3A. Assuming an equal distribution of force within the device, a $7 \mathrm{~N}$ load limit was chosen to provide $1 \mathrm{~N}$ of load per microneedle within the 7-microneedle array. A previous study by Davis et al. on the penetration force required for microneedle tissue penetration showed an approximately linear relationship between the insertion force and the interfacial area of the microneedle tip, with in vivo testing on human skin requiring insertion forces of $0.1-3 \mathrm{~N}$ for a single microneedle. ${ }^{46}$

In our study, the microneedle arrays demonstrated penetration of the cadaveric porcine skin, as evidenced by methylene blue staining of the tissue following microneedle withdrawal. Methylene blue cannot penetrate skin with an intact stratum corneum layer. On the other hand, damaged skin (with a discontinuous stratum corneum layer) allows for diffusion of methylene blue; as such, a marked color change may be observed at the site of tissue damage. ${ }^{47}$ Optical images of the methylene blue-stained tissue show three distinct puncture marks; two potential puncture marks were noted below the distinct puncture marks (Figure 3B). Two of the top puncture marks are approximately $1360 \mu \mathrm{m}$ apart; this measurement closely corresponds with the $1400 \mu \mathrm{m}$ microneedle-to-microneedle spacing of the microneedle design. The increased spacing between the other puncture marks is possibly due to the creases within the skin and the elastic nature of the tissue. The porcine hairs shown in the image potentially obstructed the other microneedles within the array, preventing penetration of the 
tissue by all of the microneedles within the array. Porcine tissue that was treated with methylene blue without microneedle application showed no distinct puncture marks; however, some discoloration of the tissue due to the dye was noted.

Evaluation of the microneedle structure and N-UNCD coating after porcine skin penetration testing was conducted using the stereomicroscope and SEM. SEM images of N-UNCD titanium microneedle array after insertion into cadaveric porcine skin show the microneedle tips remained fully intact with no N-UNCD film delamination or fracture (Figure 3C). The results suggest that the microneedle arrays are suitably robust for skin penetration and that the N-UNCD coating has sufficient adhesion to the titanium alloy substrate for skin penetration.

\subsection{Electrochemical Characterization}

N-UNCD coated titanium substrates and uncoated titanium substrates were electrochemically characterized in potassium ferricyanide using cyclic voltammograms (CV's) as seen in Figure 4A. Uncoated Ti-6Al$4 \mathrm{~V}$ alloy chips and N-UNCD coated chips run at $10 \mathrm{mV} / \mathrm{s}$ scan rate in potassium ferricyanide showed that the uncoated titanium alloy substrates possess no redox activity towards the ferricyanide solution, with no visible oxidation or reduction peaks (data not shown). The N-UNCD coated sample, run using the same parameters, showed reversible oxidative and reductive peaks.

For further characterization of the N-UNCD coatings, CV's were run from -0.1 to $0.6 \mathrm{~V}$ at scan rates varying from 10 to $300 \mathrm{mV} / \mathrm{s}$. The $\mathrm{N}-\mathrm{UNCD}$ CV's showed reversible oxidative and reductive peaks, $\Delta \mathrm{Ep}=180 \mathrm{mV}$ at 100 $\mathrm{mV} / \mathrm{s}$, indicating facile electron transfer at the diamond surface and stability over three cycles. Plots of the reduction and oxidation peak currents versus square root of the scan rate are seen in Figure 4B. A linear relationship was seen for both the reduction and oxidation peaks to the scan rate, indicating diffusion-controlled reaction, as described by the Cottrell Equation. ${ }^{48}$

These results are similar to the results of other studies that involve use of nitrogen-doped diamond electrodes for electrochemical sensing. Zhang et al. evaluated the electrochemical properties of nitrogen-doped, boron-doped, and undoped diamond. The study demonstrated characteristic CV behavior for a ferricyanide redox couple on the nitrogen-doped diamond films ${ }^{12}$. Shalini et al. also demonstrated a quasireversible response of 
deposited at several temperatures. ${ }^{25}$ This study compared the electrochemical characteristics of the nitrogendoped diamond to those of boron-doped diamond and glassy carbon; these materials demonstrated $\Delta$ Ep values of $98 \mathrm{mV}$ and $210 \mathrm{mV}$, respectively. ${ }^{25}$

\section{4 In Vitro Electrochemical Detection of Uric Acid and Dopamine using N-UNCD Electrode}

In vivo sensors enable real-time detection of physiologically relevant molecules that may aid in diagnosis, monitoring of patient health, and development of therapeutic treatments. Uric acid is produced through metabolic breakdown of purine nucleotides and is present in human blood serum and urine. At high levels, uric acid is associated with various medical conditions including gout, arthritis, diabetes, hypercholesterolemia, as well as cardiovascular and kidney diseases. ${ }^{49}$ Dopamine is a primary neurotransmitter molecule that is present in the central and peripheral nervous system. The neurotransmitter functions in the nervous system as well as the renal, hormonal, and cardiovascular systems; it has been used for investigation of neural disorders such as Parkinson's and schizophrenia. ${ }^{25}$ Both biomolecules are of considerable importance for patient health, requiring reliable and sensitive detection mechanisms.

In this study, linear scanning voltammetry was used to detect uric acid and dopamine by running scans from $-0.2 \mathrm{~V}$ to $0.8 \mathrm{~V}$ at $10 \mathrm{mV} / \mathrm{sec}$ (Figure 5). Uric acid concentrations varying from 0 to $200 \mu \mathrm{M}$ were evaluated. A distinct oxidation peak was observed at a potential of $0.48 \mathrm{~V}$ (Figure $5 \mathrm{~A}$ ). A linear relationship was observed between the uric acid concentration and the peak current throughout the detection range (Figure 5B). Oxidation peak potentials were consistent across the range of tested concentrations. Dopamine concentrations varying from 0-30 $\mu \mathrm{M}$ were detected at an oxidation peak potential of $0.65 \mathrm{~V}$ (Figure $6 \mathrm{~A}$ ). The small oxidation peak at $0.3 \mathrm{~V}$ for the $30 \mu \mathrm{M}$ concentration was due to experimental error, with no additional peaks visible on other scans. Varying concentrations of dopamine were tested from 0 to $30 \mu \mathrm{M}$ with a linear relationship seen across this range (Figure 6B). Detection above this value resulted in inaccurate readings with variable peak currents. The oxidative peaks between the two analytes were separated by $\sim 200 \mathrm{mV}$ when tested separately; however, attempts to detect the 
two analytes simultaneously were unsuccessful and only a single peaks was observed due to overlapping of the individual signals. Previous studies have demonstrated simultaneous detection of uric acid and dopamine using nitrogen-incorporated UNCD electrodes. ${ }^{25}$ Shalini et al. demonstrated simultaneous detection of dopamine, uric acid, and ascorbic acid with significant peak separation using nitrogen-doped diamond electrodes. ${ }^{25,42}$ They noted that the nitrogen-doped diamond electrodes demonstrated superior peak separation compared to the borondoped diamond, graphite, and glass carbon electrodes. ${ }^{25,42}$ A detection limit for dopamine of $0.36 \mu \mathrm{M}$ in the presence of ascorbic acid was demonstrated. ${ }^{25}$ Their results show the sensitivity and high selectivity of nitrogendoped diamond for simultaneous detection of physiologically relevant molecules. Future studies involving nitrogen-doped diamond coated microneedles will investigate varying deposition parameters and detection procedures to improve the N-UNCD electrode and further resolve the two peaks.

Although unmodified doped diamond electrodes have shown excellent capabilities for detection of physiologically relevant small molecules, the major advantage of using diamond-based electrodes lies in the potential of these electrodes for bio-functionalization. The surface of nanocrystalline diamond may be chemically functionalized to produce exceptionally tunable biomolecular interfaces. ${ }^{50}$ Developments in chemical and photochemical processes for covalent attachment of functional biomolecules have further broadened the use of NCD electrodes in advanced biosensors. Future studies will investigate the use of N-UNCD microneedle electrodes with immobilized biomolecules (e.g., enzymes and antibodies) for sensitive and highly selective electrochemical detection of biomolecules.

\section{Conclusions}

We have demonstrated successful fabrication of nitrogen-incorporated ultrananocrystalline diamondcoated titanium alloy microneedle arrays that are capable of electrochemical detection of dopamine and uric acid. The N-UNCD coated microneedle arrays were fabricated in a straightforward and reproducible manner; minimal microneedle-to-microneedle variation in microneedle dimensions was noted. The N-UNCD coating deposited by CVD was continuous with small grain sizes and exhibited the typical carbon bonding signatures representative of $\mathrm{N}$ UNCD thin films. Diamond-coated titanium microneedle arrays penetrated full-thickness cadaveric porcine skin without microneedle fracture or diamond film delamination, demonstrating suitable robustness for transdermal 
applications. Electrochemical analysis of the N-UNCD coatings on the titanium alloy show suitable electron transfer rates and reversibility for electrochemical biosensing applications. In vitro electrochemical detection of uric acid and dopamine using unmodified N-UNCD electrodes were observed across physiologically relevant concentrations for each analyte. By utilizing the superior electrochemical properties of N-UNCD as well as its potential for biofunctionalization, we envision the use of these N-UNCD-coated titanium alloy microneedle arrays for many types of transdermal electrochemical biosensing applications.

\section{Acknowledgements:}

One of the authors (SAS) was supported in part by NSF Award \#936110. Use of the Center for Nanoscale Materials was supported by the U. S. Department of Energy, Office of Science, Office of Basic Energy Sciences, under Contract No. DE-ACO2-06CH11357. 


\section{References}

[1] J.R. Windmiller, G. Valdes-Ramirez, N. Zhou, M. Zhou, P.R. Miller, C. Jin, S.M. Brozik, R. Polsky, E. Katz, R. Narayan, J. Wang, Biocomponent microneedle array biosensor for minimally-invasive glutamate monitoring, Electroanalysis 23 (2011) 2302-2309. DOI: 10.1002/elan.201100361

[2] P.R. Miller, S.D. Gittard, T.L. Edwards, D.M. Lopez, X. Xiao, D.R. Wheeler, N.A. Monteiro-Riviere, S.M. Brozik, R. Polsky, R.J. Narayan, Integrated carbon fiber electrodes within hollow polymer microneedles for transdermal electrochemical sensing, Biomicrofluidics 5 (2011) 013415. DOI:10.1063/1.3569945

[3] E.V. Mukerjee, S.D. Collins, R.R. Isseroff, R.L. Smith, Microneedle array for transdermal biological fluid extraction and in situ analysis, Sensors and Actuators A: Physical 114 (2004) 267-275. DOI:

10.1016/j.sna.2003.11.008

[4] M.A. Invernale, B.C. Tang, R.L. York, L. Le, D.Y. Hou, D.G. Anderson, Microneedle electrodes toward an amperometric glucose-sensing smart patch, Advanced Healthcare Materials 3 (3) (2014) 338-42. DOI: 10.1002/adhm.201300142

[5] B. Chua, S.P. Desai, M.J. Tierney, J.A. Tamada, A.N. Jina, Effect of microneedles shape on skin penetration and minimally invasive continuous glucose monitoring in vivo, Sensors and Actuators A 203 (2013) 373-381. DOI: 10.1016/j.sna.2013.09.026

[6] Y. Yoon, G.S. Lee, K. Yoo, J.B. Lee, Fabrication of a microneedle/CNT hierarchical micro/nano surface electrochemical sensor and its in-vitro glucose sensing characterization, Sensors 13 (2013) 16672-16681. DOI:10.3390/s131216672

[7] J.R. Windmiller, N. Zhou, M.C. Chuang, G. Valdes-Ramirez, P. Santhosh, P.R. Miller, R. Narayan, J. Wang, Microneedle array-based carbon paste amperometric sensors and biosensors, Analyst 136 (2011) 1846-1851. DOI: 10.1039/c1an00012h

[8] P.R. Miller, S.A. Skoog, T.L. Edwards, D.M. Lopez, D.R. Wheeler, D.C. Arango, X. Xiao, S.M. Brozik, J. Wang, R. Polsky, R.J. Narayan, Talanta 88 (2011) 739-42. DOI: 10.1016/j.talanta.2011.11.046

[9] A. Qureshi, W.P. Kang, J.L. Davidson, Y. Gurbuz, Review on carbon-derived, solid-state, micro and nano sensors for electrochemical sensing applications, Diamond \& Related Materials 18 (2009) 14011420. DOI:10.1016/j.diamond.2009.09.008

[10] Z. Xu, A. Kumar, A. Kumar, Amperometric detection of glucose using modified nitrogen-doped nanocrystalline diamond electrode, Journal of Biomedical Nanotechnology, 1 (2005) 416-420.

DOI:10.1166/jbn.2005.057

[11] R.J. Narayan, R.D. Boehm, A.V. Sumant, Medical applications of diamond particles \& surfaces, Materials Today 14(4) (2011) 154-163. DOI: 10.1016/S1369-7021(11)70087-6. 
[12] Y. Zhang, S. Yoshihara, T. Shirakashi, T. Kyomen, Electrochemical characteristics of boron-doped, undoped, and nitrogen-doped diamond films, Diamond \& Related Materials 14 (2005) 213-219.

DOI:10.1016/j.diamond.2004.11.039

[13] A Hartl, E. Schmich, J.A. Garrido, J. Hernando, S.C.R. Catharino, S. Walter, P. Feulner, A. Kromka, D. Steinmuller, M. Stutzmann, Protein-modified nanocrystalline diamond thin films for biosensor applications, Nature Materials 3 (2004) 736-742. DOI:10.1038/nmat1204

[14] J. Rubio-Retama, J. Hernando, B. Lopez-Ruiz, A. Hartl, D. Steinmuller, M. Stutzmann, E. LopezCabarcos, J.A. Garrido, Synthetic nanocrystalline diamond as a third-generation biosensor support, Langmuir 22 (2006) 5837-5842. DOI:

[15] C.E. Nebel, D. Shin, B. Rezek, N. Tokuda, H. Uetsuka, H. Watanabe, Diamond and biology, Journal of the Royal Society Interface 4 (2007) 439-461. DOI:10.1098/rsif.2006.0196

[16] J.H.T. Luong, K.B. Male, J.D. Glennon, Boron-doped diamond electrode: synthesis, characterization, functionalization and analytical applications, Analyst 134 (2009) 1965-1979. DOI:10.1039/b910206j

[17] J. Hees, R. Hoffman, A. Kriele, W. Smirnov, H. Obloh, K. Glorer, B. Raynor, R. Driad, N. Yang, O.A. Williams, C.E. Nebel, Nanocrystalline diamond nanoelectrode arrays and ensembles, ACS Nano 5(4) (2011) 3339-3346. DOI: 10.1021/nn2005409

[18] J.W. Strojek, M.C. Granger, G.M. Swain, Enhanced signal-to-background ratios in voltammetric measurements made at diamond thin-film electrochemical interfaces, Analytical Chemistry 68(13) (1996) 2031-2037. DOI:10.1021/ac9506847

[19] C. Cofan, C. Radovan, Simultaneous chronoamperometric sensing of ascorbic acid and acetaminophen at a boron-doped diamond electrode, Sensors 8 (2008) 3952-3969. DOI: $10.3390 / \mathrm{s} 8063952$

[20] S. Xie, G. Shafer, C.G. Wilson, H.B. Marin, In vitro adenosine detection with a diamond-based sensor, Diamond \& Related Materials 15 (2006) 225-228. DOI:10.1016/j.diamond.2005.08.018

[21] M.C. Granger, J. Xu, J.W. Strojek, G.M. Swain, Polycrystalline diamond electrodes: basic properties and applications as amperometric detectors in flow injection analysis and liquid chromatography, Analytica Chimica Acta 397 (1999) 145-161. DOI: 10.1016/S0003-2670(99)00400-6

[22] N. Spataru, B.V. Sarada, D.A. Tryk, A. Fujishima, Anodic voltammetry of xanthine, theophylline, theobromine and caffeine at conductive diamond electrodes and its analytical application, Electroanalysis 14(11) (2002) 721-728. DOI:040-0397/02/1106-0721

[23] O. Chailapakul, E. Popa, H. Tai, B.V. Sarada, D.A. Tryk, A. Fujishima, The electrooxidation of organic acids at boron-doped diamond electrodes, Electrochemistry Communications 2 (2000) 422-426. 
[24] A. Fujishima, T.N. Rao, E. Popa, Electroanalysis of dopamine and NADH at conductive diamond electrodes, Journal of Electroanalytical Chemistry and Interfacial Electrochemistry 473 (1999) 179. DOI: 10.1016/S0022-0728(99)00106-0

[25] J. Shalini, K.J. Sankaran, C.L. Dong, C.Y. Lee, N.H. Tai, I.N. Lin, In situ detection of dopamine using nitrogen incorporated diamond nanowire electrode, Nanoscale 5 (2013) 1159-1167. DOI:

$10.1039 / c 2 n r 32939 e$

[26]O. Chailapakul, P. Aksharanandana, T. Frelink, Y. Einaga, A. Fujishima, The electrooxidation of sulfurcontaining compounds at boron-doped diamond electrode, Sensors and Actuators B: Chemical 80 (3) (2001) 193-201. DOI: 10.1016/S0925-4005(01)00912-1

[27] B.V. Sarada, T.N. Rao, D.A. Tryk, A. Fujishima, Electrochemical oxidation of histamine and serotonin at highly boron-doped diamond electrodes, Analytical Chemistry 72 (2000) 1632-1638.

DOI:10.1021/ac9908748

[28] T.A. Ivandini, T.N. Rao, A. Fujishima, Y. Elnaga, Electrochemical oxidation of oxalix acid at highly boron-doped diamond electrodes, Analytical Chemistry 78 (2006) 3467-3471. DOI:10.1021/ac052029x

[29] J.M. Halpern, S. Xie, G.P. Sutton, B.T. Higashikubo, C.A. Chestek, H. Lu, H.J. Chiel, H.B. Martin, Diamond electrodes for neurodynamic studies in Aplysia californica, Diamond \& Related Materials 15 (2006) 183-187. DOI:10.1016/j.diamond.2005.06.039

[30] W. Yang, O. Auciello, J.E. Butler, W. Cai, J.A. Carlisle, J. Gerbi, D.M. Gruen, T. Knickerbocker, T.L. Lasseter, J.N. Russell, L.M. Smith, R.J. Hamers, DNA-modified nanocrystalline diamond thin-films as stable, biologically active substrates, Nature Materials 1 (2002) 253-257. DOI:10.1038/nmat779

[31] J. Wang, Electrochemical glucose biosensors, Chemical Reviews 108 (2008) 814-825.

[32] C.N. Elias, J.H.C. Lima, R. Valiev, M.A. Meyers, Biomedical applications of titanium and its alloys, JOM (2008) 46-49.

[33] C.Z. Zhang, Y.S. Li, Y. Tang, L. Yang, L. Zhang, Y. Sun, Q. Yang, A. Hirose, Nanocrystalline diamond thin films grown on Ti6Al4V alloy, Thin Solid Films 527 (2013) 59-64. DOI:10.1016/j.tsf.2012.12.014

[34] N. Roxhed, T.C. Gasser, P. Griss, G.A. Holzapfel, G. Stemme, Penetration-enhanced ultrasharp microneedles and prediction on skin interaction for efficient transdermal drug delivery, Journal of Microelectromechanical Systems 16 (2007) 1429-1440. DOI: 10.1109/JMEMS.2007.907461

[35] S. Wenmackers, S.D. Pop, K. Roodenko, V. Vermeeren, O.A. Williams, M. Daenen, O. Douheret, J. D'Haen, A. Hardy, M.K. Van Bael, K. Hinrichs, C. Cobet, M. vandeVen, M. Ameloot, K. Haenen, L. Michiels, N. Esser, P. Wagner, Structural and optical properties of DNA layers covalently attached to diamond surfaces, Langmuir 24 (2008) 7269-7277. DOI: 10.1021/la800464p 
[36] A. Hartl, E. Schmich, J.A. Garrido, J. Hernando, S.C.R. Catharino, S. Walter, P. Fuelner, A. Kromka, D. Steinmuller, M. Stutzmann, Protein-modified nanocrystalline diamond thin films for biosensor applications, Nature Materials 3 (2004) 736-742. DOI: 10.1038/nmat1204

[37] C. Stavis, T.L. Clare, J.E. Butler, A.D. Radadia, R. Carr, H. Zheng, W.P. King, J.A. Carlisle, A. AKsimentiev, R. Bashir, R.J. Hamers, Surface functionalization of thin-film diamond for highly stable and selective biological interfaces, Journal of the American Chemical Society 108 (2010) 983-988. DOI/10.1073/pnas.1006660107

[38] W. Yang, O. Auciello, J.E. Butler, W. Cai, J.A. Carlisle, J. Gerbi, D.M. Gruen, T. Knickerbocker, T.L. Lasseter, J.N. Russell, L.M. Smoth, R.J. Hamers, DNA-modified nanocrystalline diamond thin-films as stable, biologically active substrates, Nature Materials 1 (2002) 253-257. doi:10.1038/nmat779

[39] A. Kraft, Doped diamond: a compact review on a new, versatile electrode material, International Journal of Electrochemical Science 2 (2007) 355-385.

[40] S. Bhattacharyya, O. Auciello, J. Birrell, J.A. Carlisle, L.A. Curtiss, A.N. Goyette, D.M. Gruen, A.R. Krauss, J. Schlueter, A. Sumant, P. Zapol, Synthesis and characterization of highly-conducting nitrogendoped ultrananocrystalline diamond films, Applied Physics Letters 79(10) (2001) 1441. DOI: 10.1063/1.1400761.

[41] K.J. Sankaran, J. Kurian, H.C. Chen, C.L. Dong, C.Y. Lee, N.H. Tai, I.N. Lin, Origin of a needle-like granular structure for ultrananocrystalline diamond films grown in a N2/CH4 plasma, Journal of Physics D: Applied Physics 45 (2012) 365303. DOI:10.1088/0022-3727/45/36/365303

[42] J. Shalini, Y.C. Lin, T.H. Chang, K.J. Sankaran, H.C. Chen, I.N. Lin, C.Y. Lee, N.H. Tai, Ultrananocrystalline diamond nanowires with enhanced electrochemical properties, Electrochimica Acta 92 (2013) 9-19. DOI:10.1016/j.electacta.2012.12.078

[43] W. Kulisch, C. Popov, H. Rauscher, M. Rinke, M. Veres, Investigation of the initial growth of ultrananocrystalline diamond films by multiwavelength Raman spectroscopy, Diamond \& Related Materials 20 (2011) 1076-1080. DOI:10.1016/j.diamond.2011.03.042

[44] I.I. Vlasov, V.G. Ralchenko, E. Goovaerts, A.V. Saveliev, M.V. Kanzyuba, Bulk and surface-enhanced Raman spectroscopy of nitrogen-doped ultrananocrystalline diamond films, physica status solidi A 203 (12) (2006) 3028-3035. DOI 10.1002/pssa.200671119

[45] J. Birrell, J.E. Gerbi, O. Auciello, J.M. Gibson, D.M. Gruen, J.A. Carlisle, Bonding structure in nitrogen doped ultrananocrystalline diamond, Journal of Applied Physics 93 (2003) 5606. DOI:

10.1063/1.1564880

[46] S.P. Davis, B.J. Landis, Z.H. Adams, M.G. Allen, M.R. Prausnitz, Insertion of microneedles into skin: measurement and prediction of insertion force and needle fracture force, Journal of Biomechanics 37 (2004) 1155-1163. DOI:10.1016/j.jbiomech.2003.12.010 
[47] R.F. Donnelly, R. Majithiya, T.R.R. Singh, D.I.J. Morrow, M.J. Garland, Y.K. Demir, K. Migalska, E. Ryan, D. Gillen, C.J. Scott, A.D. Woolfson, Design, optimization and characterization of polymeric microneedle arrays prepared by a novel laser-based micromoulding technique, Pharmaceutical Research 28 (2011) 41-57. DOI 10.1007/s11095-010-0169-8

[48] Y. Harek, L. Larabi, L. Boukli, F. Kadr, N. Benali-Cherif, M. M. Mostafa, Synthesis, characterization, crystal structure and electrochemical behavior of a nickel(II) complex of 5,6-dihydro-2H-pyran-3aldehyde thiosemicarbazone, Transition Metal Chemistry 30 (2005) 121-127. DOI: 10.1007/s11243-0041565-x.

[49] K. Jindal, M. Tomar, V. Gupta, Inducing electrocatalytic functionality in ZnO thin film by N doping to realize a third generation uric acid biosensor, Biosensors and Bioelectronics 55 (2004) 57-65. DOI:

10.1016/j.bios.2013.11.015

[50] C. Stavis, T.L. Clare, J.E. Butler, A.D. Radadia, R. Carr, H. Zeng, W.P. King, J.A. Carlisle, A. Aksimentiev, R. Bashir, R.J. Hamers, Surface functionalization of thin-film diamond for highly stable and selective biological interfaces, Proceedings of the National Academy of Sciences USA 108(3) (2011) 983-988. DOI:10.1073/pnas.1006660107 
Figure 1. SEM images of uncoated titanium alloy microneedle arrays (A-C) and N-UNCD coated arrays (DF). High magnification images show the surface morphologies of the uncoated titanium alloy microneedles $(C)$ and the N-UNCD coated microneedles (F).

Figure 2. Raman spectra of the N-UNCD coating on titanium alloy disk, microneedle tip (MN tip), and flat base (MN flat) of microneedle array.

Figure 3. Skin penetration test setup (A). Optical micrographs of full-thickness cadaveric porcine skin stained with methylene blue after penetration by a microneedle array (B). SEM micrograph of N-UNCD titanium microneedle array tip after insertion into cadaveric porcine skin (C)

Figure 4. Cyclic voltammetric scans of potassium ferricyanide in $1 X \mathrm{PBS} p \mathrm{pH} 7.4$ versus a $\mathrm{Ag} / \mathrm{AgCl}$ reference and Pt wire counter electrode at varying scan rates from $10-300 \mathrm{mV} / \mathrm{s}(\mathrm{A})$ and plots of the reduction and oxidation peak currents versus square root of the scan rate (B).

Figure 5. Linear sweep voltammograms of N-UNCD electrodes in a $1 \mathrm{X}$ PBS pH 7.4 solutions containing uric acid concentrations ranging from 0 to $200 \mu \mathrm{M}$ versus an $\mathrm{Ag} / \mathrm{AgCl}$ reference and Pt wire counter electrode (A) and a plot of uric acid oxidation peak currents at various concentrations (B).

Figure 6. Linear sweep voltammograms of N-UNCD electrodes in a 1X PBS solution containing dopamine concentrations ranging from 0 to $30 \mu \mathrm{M}$ versus an $\mathrm{Ag} / \mathrm{AgCl}$ reference and Pt wire counter electrode $(\mathrm{A})$ and a plot of the dopamine oxidation peak currents at various concentrations (B). 
Figure 1.
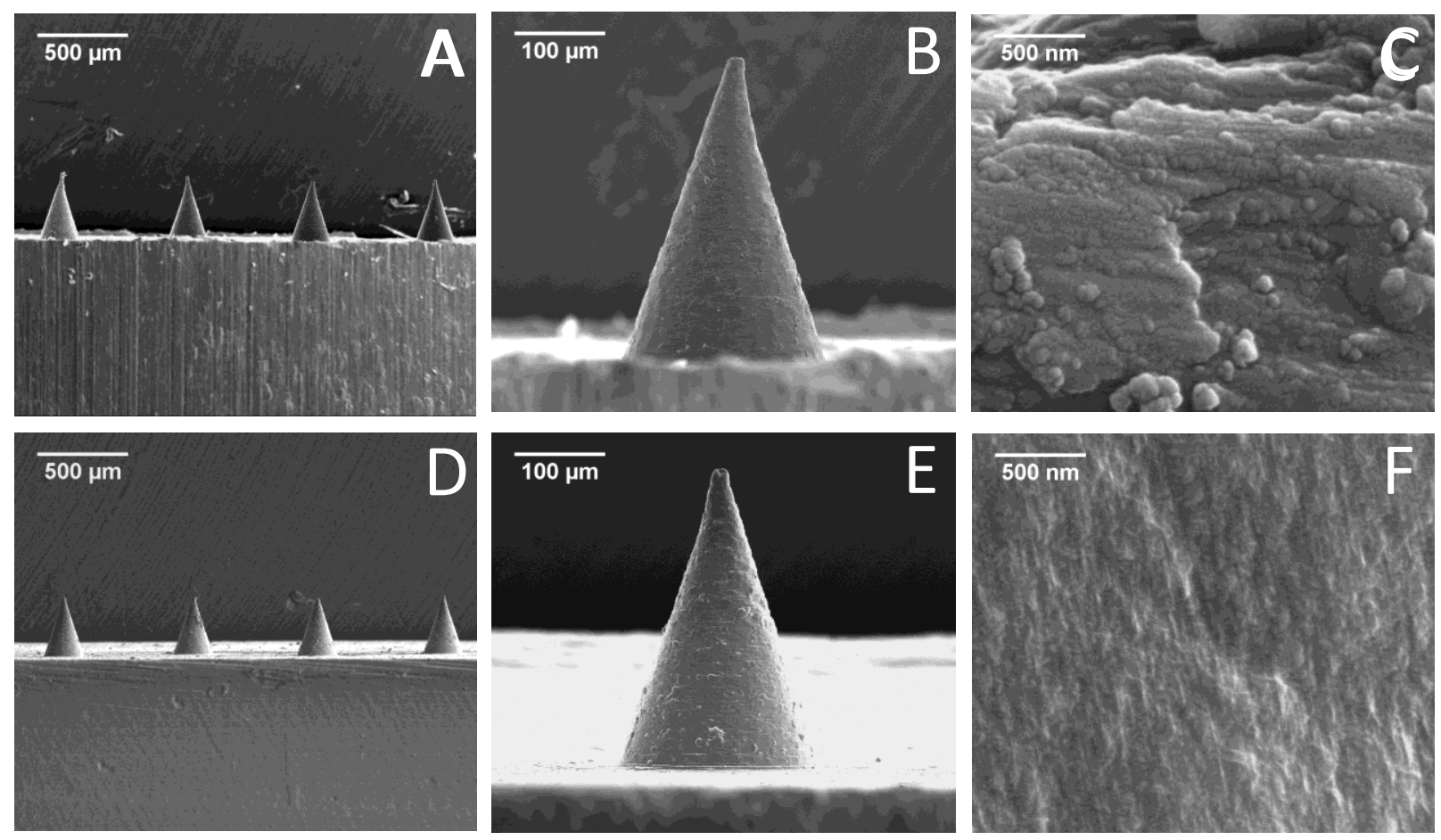

32

33

34

35

36

37

38

39

40

41

42

43

44

45

46

47

48

49

50

51

52

53

54

55

56

57

58

59 
Figure 2

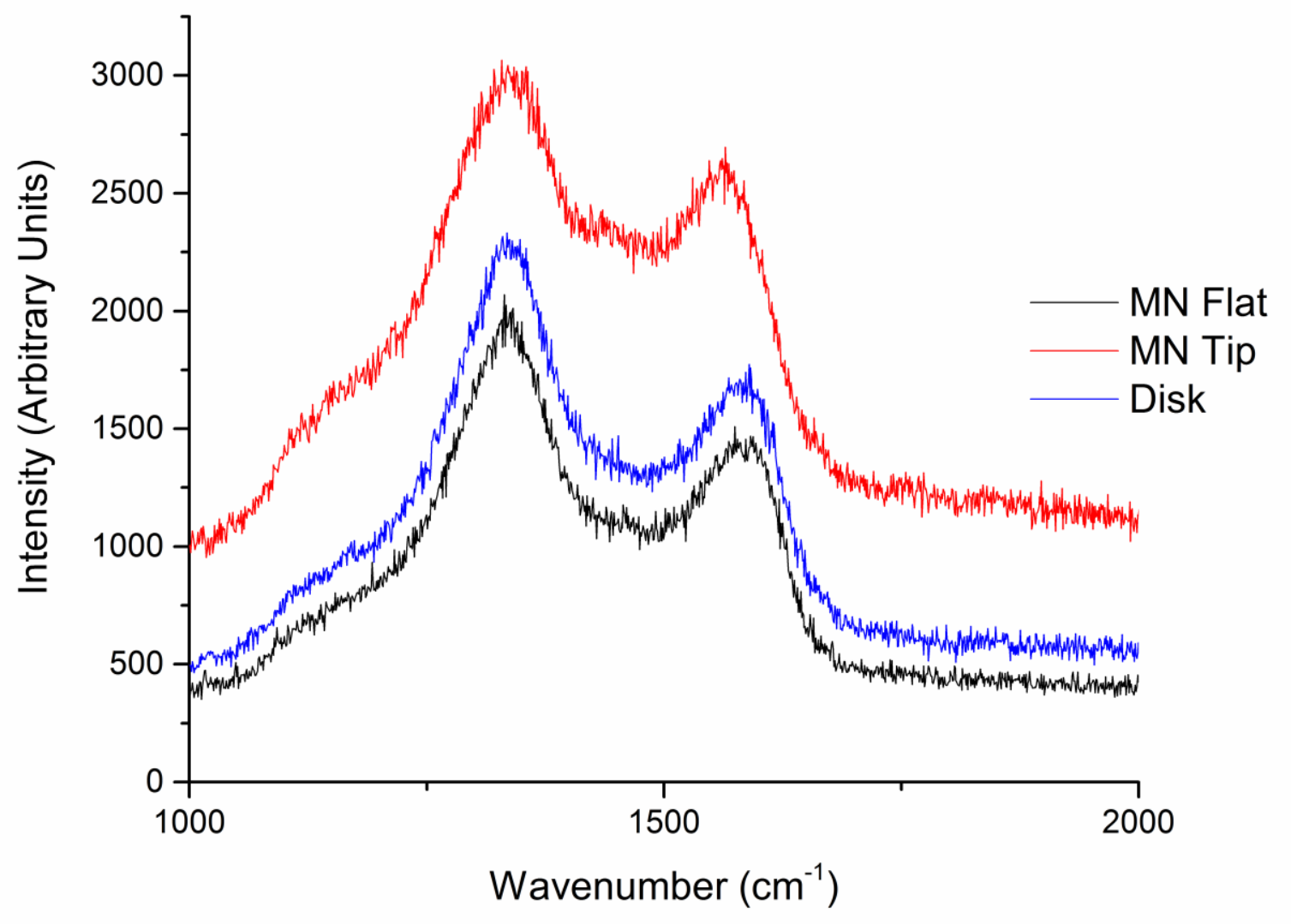


Figure 3

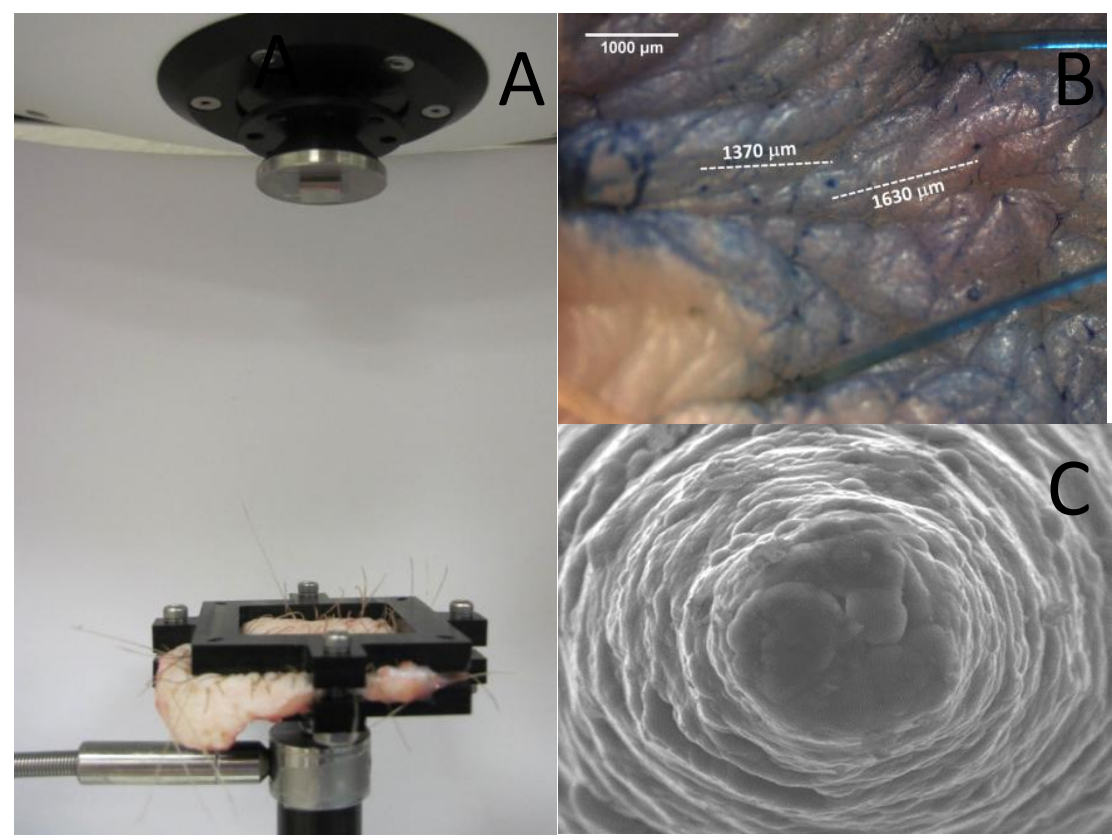

33

34

35

36

37

38

39

40

41

42

43

44

45

46

47

48

49

50

51

52

53

54

55

56

57

58

59

60

61

62

63 
Figure 4

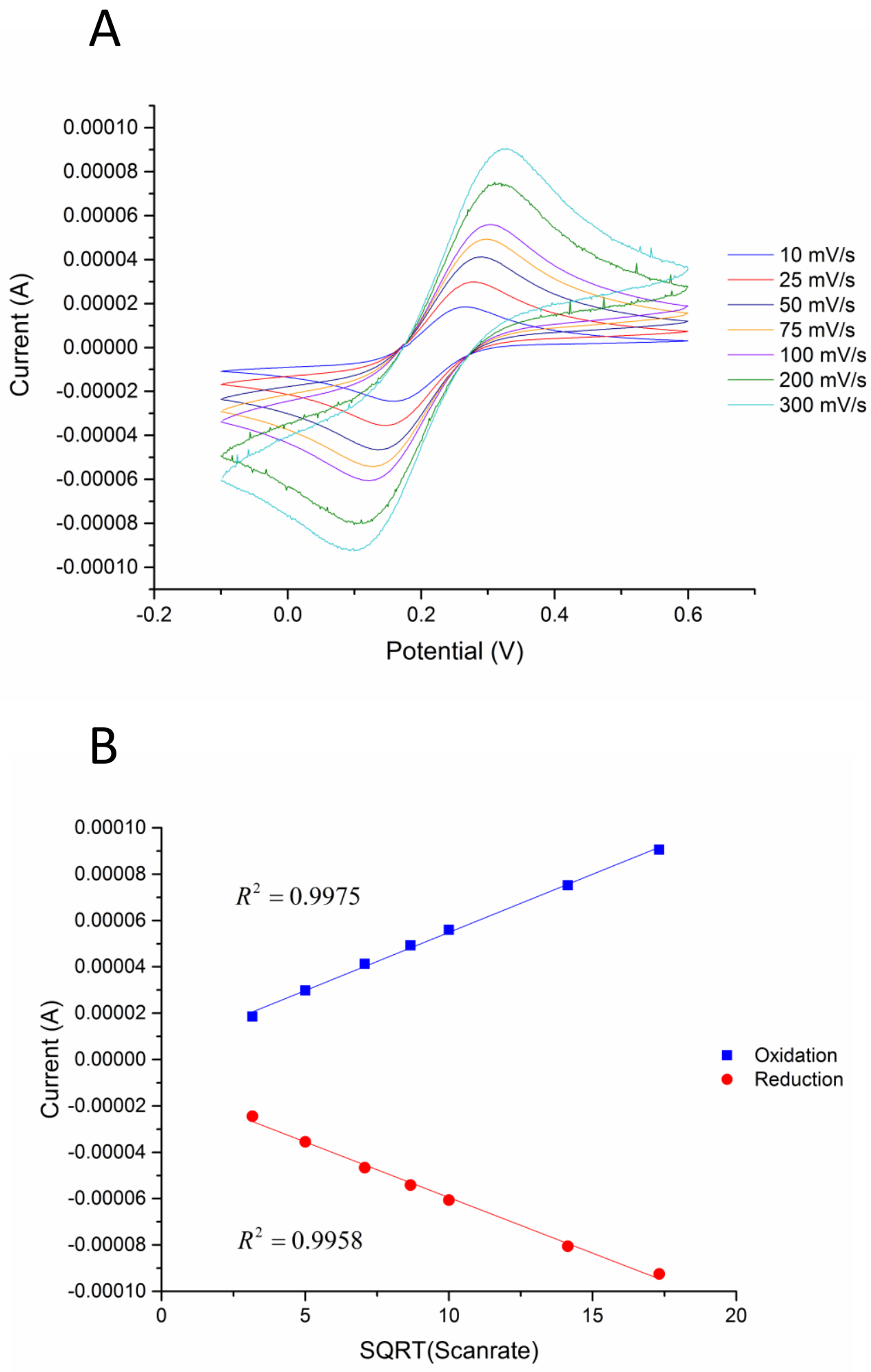


Figure 5
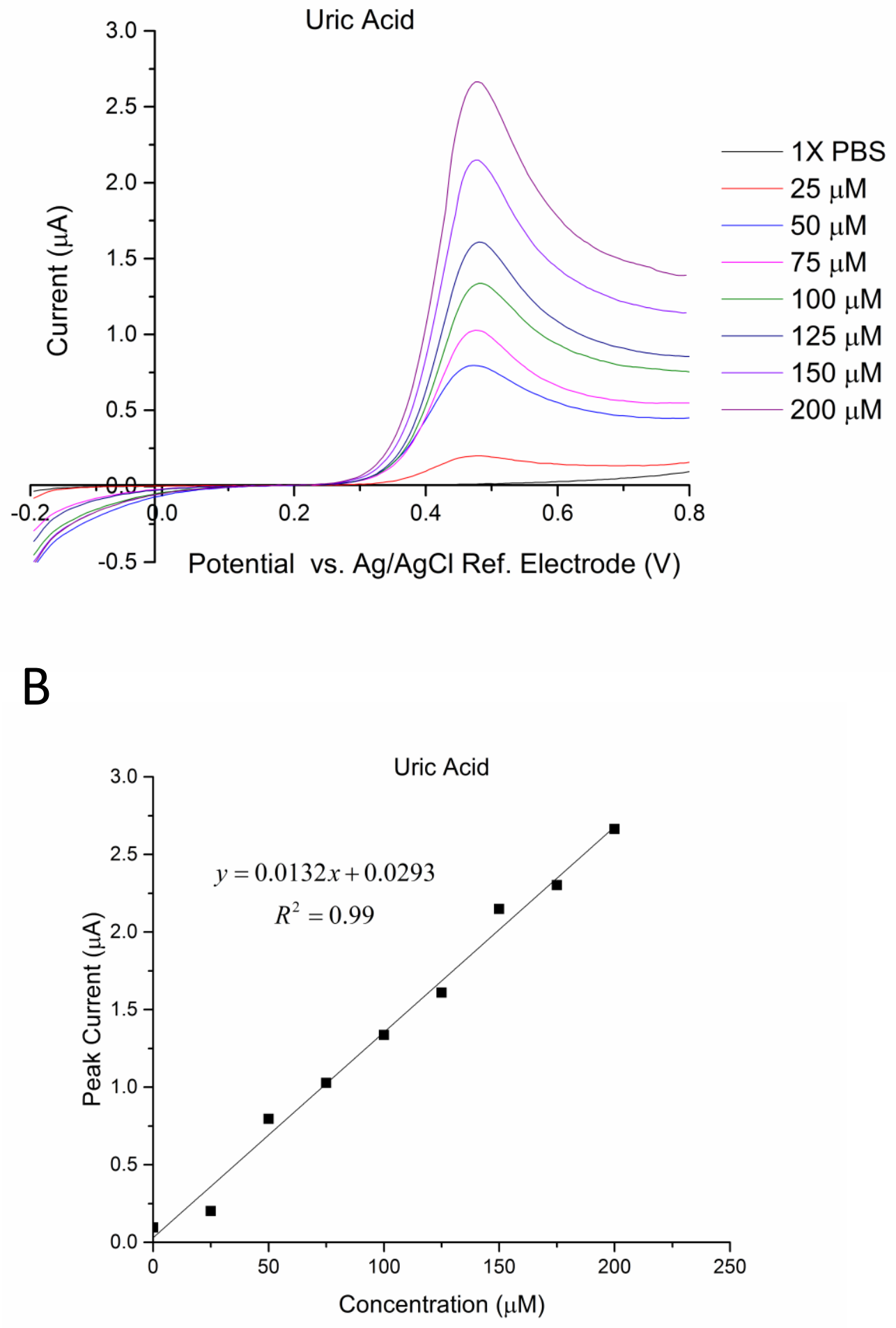

59

60

61

62

63 
Figure 6

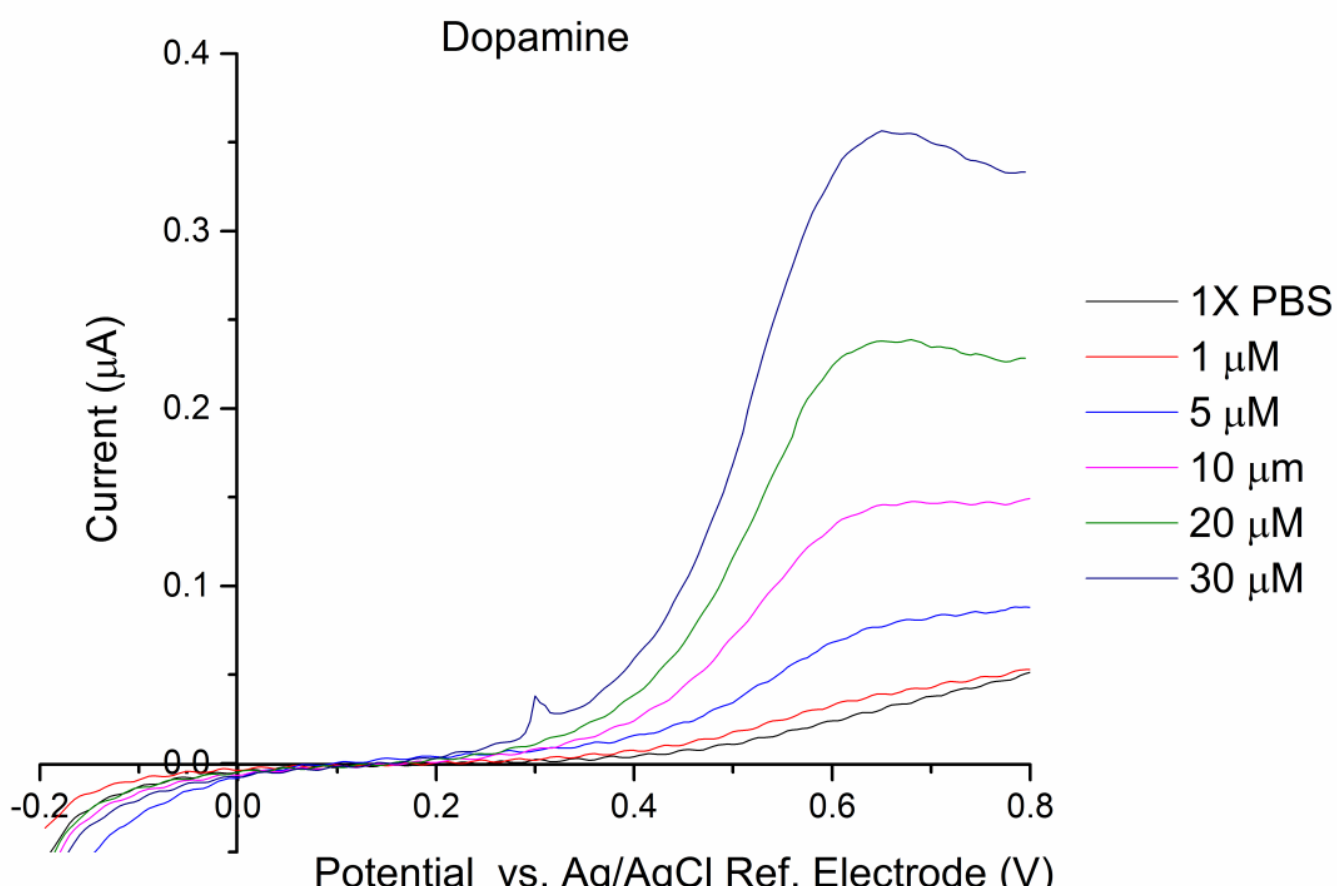

33

Potential vs. Ag/AgCl Ref. Electrode (V)

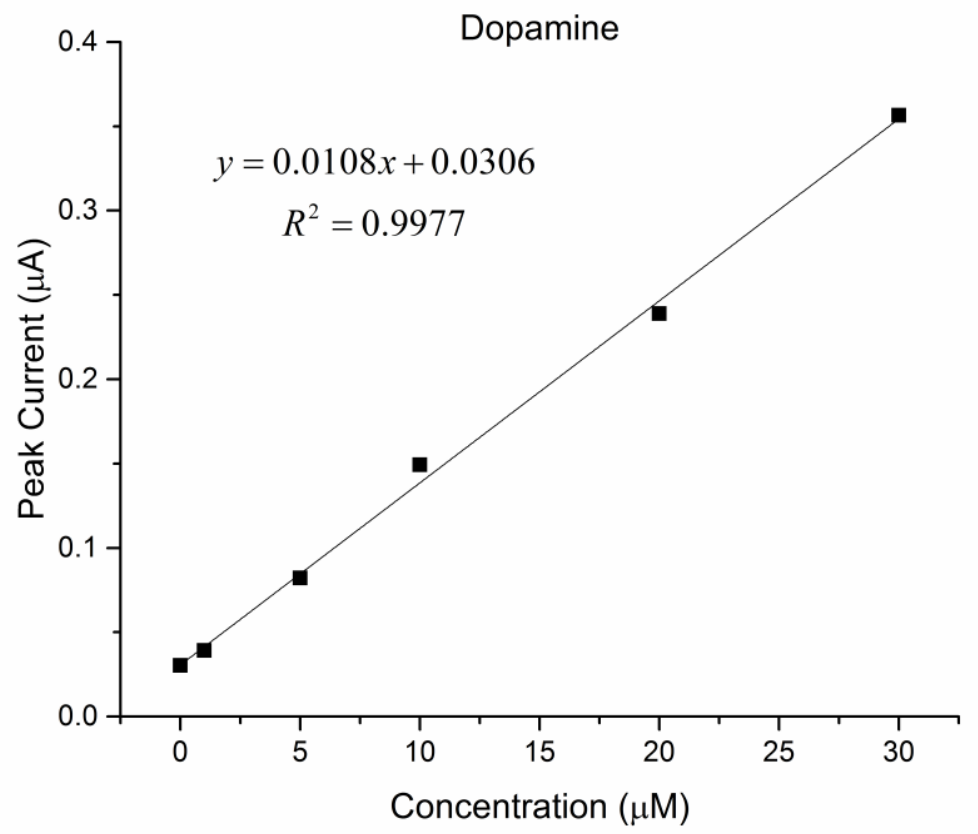

